\title{
Addendum
}

\section{Conclusions of the SAPT Sessions}

\begin{abstract}
The following presents the conclusions gathered from the presentations, posters and discussions of the SAPT sessions. They are presented on the animal, feed resources and system levels.
\end{abstract}

On the animal level, the importance of choosing the right breed for the right environment was stated. Therefore local tropical animal genetic resources must be characterized; tropical countries own a large variety of local breeds, with specific adaptation traits, that could be of major importance for the development of livestock production systems in a changing world. Sustainability of livestock production in the tropics relies on this diversity. In addition, Genotype*Environment Interactions (GEI) appear to have a major importance for livestock breeding and genetics in the tropics. Interest in this field will probably increase in the future, along with more frequent international exchanges and the growing concern for animal production at a global level. The consequences of GEl on animal genetic resource management were described for the Australian experience. The incidence of GEI on genomic selection appears as a great challenge for research and its application in the future. Australian scientists have a great experience in this field. However, it seems that collaborative programs involving various countries and production systems, and different genotypes or breed types may tackle this challenge.

The discovery of QTL and causal mutations associated with adaptation to tropical environments is a worthy goal, but based on previous reports in the scientific literature, the chances of success are not high, so less risky approaches (e.g. genomic selection) should not be ignored if the aim is to achieve solutions for farmers rather than just reports in the scientific literature. Traditional approaches to breeding programs (e.g. crossbreeding and within-breed improvement where feasible) still have a lot of potential to improve productivity of farms in the tropics.

Sustainability of tropical breeding systems must be strengthened by multidisciplinary approaches (physiology and animal genetics). Efforts to identify new, easily measured on-farm ways of identifying animals that are resistant or tolerant to stressors of tropical environments, on large numbers of animals, possibly under difficult field conditions, would be well justified. Heat stress, nutritional stress and pathologies were addressed.

Climatic factors are among the main limiting factors for the development of animal production in warm regions. Effective strategies to alleviate heat stress can be classified into three groups, those increasing feed intake or decreasing metabolic heat production, those enhancing heat loss capacities, and those involving genetic selection for heat-tolerance. While differences in thermal-tolerance exist between livestock species, there are also large differences between breeds of a species and within each breed. Consequently, the opportunity may exist to improve thermal tolerance of the animals using genetic tools.

Infectious diseases of tropical livestock are critically important and need to be controlled or managed. There are a wide variety of short-term and long-term control strategies available, depending upon the disease. Disease eradication is, in many cases, the most desirable option but it requires an effective vaccine along with the collective will of many agencies involved in disease control in several countries. For many endemic diseases, control will be best achieved through a mixture of short-term and long-term control measures. Nematode parasite infections in ruminants (and to a lesser extent in pigs and poultry) are good examples. Nutritional strategies can alleviate short term problems, strategic anthelmintic drenching can treat animals that are still affected, and selection can help achieve long-term control. Research efforts should be targeted to identifying complementary control strategies, which should enable more effective disease management. An interesting option that was raised at the conference, but not discussed to any extent is self-medication. For example, giving livestock farmers the option of selecting for plants with medicinal (e.g. anthelmintic) properties when necessary was suggested. For longer-term control, genetic selection of more-resistant hosts may often be a feasible partial disease management strategy.

On the feed resources level, local feed resources cannot be by-passed and have to be deeply evaluated for feeding tropical livestock. The availability of feed for livestock and the competition with human consumption in the present world were described. This food-feed competition was mainly explained by an increase in consumption of animal based-products in connection to a change in dietary patterns of people living in developing countries. The use of alternative feed resources could avoid the competition between animals and humans for cereal grains. Tropical countries have the advantage of having a great potential of new nonconventional feed resources which can be made available throughout the year, and which can be processed in a way that makes them suitable for non-ruminant animals such as pigs and poultry. The knowledge of the nutritive value of these local resources is a major constraint for development 
of animal production in tropical countries. Tropical leaves could be interesting sources of proteins for pigs especially in integrated small-scale farming systems, where the access to common protein ingredients is reduced mainly because of their increasing cost. Some legumes such as Cassia Tora or Leuceana leucocephala could be included in diets for adult indigenous chickens, without any negative consequences on growth performance. Among all the potential non conventional protein sources, worm meal can totally replace cow meat meal in the finishing diets of broilers and Morenga Oliefera seed and its by-product appeared to be a good alternative for the replacement of common protein ingredients both in ruminants and monogastric species.

Exploration and design of alternative feeding systems for ruminant livestock in the tropics by integrative system approaches were proposed. New, simple technology is needed to valorize a priori poor forage or byproducts. For example, Cuban knowledge on mixed silage (pineapple, citrus as wet byproducts and grass hay, bean straw, sugarcane bagasse as dry byproducts) and meal from dehydrated foliages of trees was exposed. Shrubby hedges are used in Zimbabwe to feed goats but technology (wood ash) is necessary to reduce the antinutritional factors that decrease intake. In Burkina Faso, mango peel and seed kernel are valorized in sheep nutrition. Finally, characterization of tropical feed resource biodiversity remains a hot spot of research. Statistical and mathematical modeling approaches are being developed to elaborate feed nutritive value tables based on large databases under different production conditions.

Ruminant production in the tropics is mainly based on pasture. For $87 \%$ of them, it is natural with a highly variable productivity and a great multifunctionality. Various strategies for improved pasture management were analyzed. They are based on stocking rate, fertilization, pasture regrowth, intensity and duration of grazing and are assessed with very different criteria (weight gain, digestibility, feeding behaviour or intake, difficult to measure at pasture). It is still difficult to provide general rules for improved management of pastures, because of local conditions and the diversity of methods used. Useful criteria are still needed, to better assess the pastoral system, and decide the appropriate rules of management that could be re-evaluated and adapted by stockbreeders. Recent advances in technology for measuring effective management criteria were presented.

Tropical production systems could be significantly improved in terms of sustainable intensification. This sustainability relies on feed resource biodiversity and must be strengthened by multidisciplinary approaches (multicriteria evaluation). Environmental sustainability (footprint carbon emissions) is improved through integrated livestock - crop forestry production systems. Chemistry of secondary compounds from tropical plants, mathematical modeling and data mining from already existing feedstuff databases were described as powerful tools for the generation of new technologies. In addition, the participation of farmers, decision makers and scientists is necessary to better define the goals of research integrating crop, livestock and forestry production systems. Finally, as a comment, the establishment of international partnerships with industrialized and developing country research institutions would efficiently provide the resources and competencies necessary to more rapidly provide those new needed technologies.

On the system level, the importance of mixed crop livestock systems in the world was pointed out and especially in the South. There is a large variety of mixed farming systems that provide $50 \%$ of the world's cereals and food consumed by poor people. The necessity to improve the productivity of such systems and the need to "re-think" the development of livestock systems while integrating environmental, social and economic sustainability constraints has been developed, particularly in developing countries with a focus on the Caribbean. This conceptual framework was illustrated by several examples of tropical livestock farming systems, in which the utilization of local resources (crops and livestock), the characterization of some tropical livestock systems, and the requirements, challenges and opportunities for organic livestock systems in tropical areas were highlighted. Agroforestry practices also guarantee the system sustainability in an agroecological approach.

The "Let a Gogo" (Milk in abundance) initiative in Haiti was important to achieve since it has multiple objectives (increased milk production, support and income for farmers, farmer training, product processing and marketing). In addition, the domestication process of neotropical animals was highlighted: integration of the animal into human culture, animals kept forcefully and dependant on humans, animals being able to breed in captivity, desirable traits to be defined. Wildlife can be exploited to sustain the future growth of the world's meat production. A guide to the use of sugarcane and its by products as animal feed, a manual for farmers and livestock production specialists (Archimède and Garcia, 2010), was launched. An original point of view on the place and the role of breeding in carbon sequestration processes in tropical grassland was shared.

The measure of the success of integrated systems was addressed. Systemic approaches aimed at developing a holistic analysis for works in the field of animal science is needed. In developing the production systems, the biotechnical (animal) aspects have to be considered as well as the human aspects. The following also need to be integrated: the farm, agricultural sector, human values, and environment. These complex systems are all labor costly and mechanization raises specific problems and constraints in relation to fossil energy. The development of participatory approaches is needed to collectively manage local resources. The way of how legislation can constrain or enhance integrated farming systems was discussed.

In the future, scales for problem emergence and problem resolution will have to be apprehended simultaneously. It is essential to integrate long-term observations. Research has to promote interdisciplinary approaches combining animal science and social and economic sciences (I.s. economics, political science, management science etc.) to better provide analytical grids to envision plausible or desirable levels of decision. 
Methodological support is needed to foster applied research and/or research for action in order to favor in situ decision processes. The importance of developing models to integrate knowledge from analytical research and explore new scenarios was stated. Besides modeling, conceiving systems may be done by « experts » from observations, analyzing what already exists and conceiving empirical experiments. "System" experiments also include creating pilot systems and the analysis of methods developed on farms.
Finally, the importance of maintaining and supporting in situ tropical research livestock production was stated. Emphasis was put on the quality and the intensity of the discussions during the congress. The willingness of the organizing committee to renew this meeting was well received by the audience. The next meeting is scheduled in 2013 during the EAAP meeting in France.

Nathalie Mandonnet 